\title{
Eficácia do Herbicida Amicarbazone Aplicado sobre a Palha ou no Solo no Controle de Plantas Daninhas na Cultura da CANA-DE-AÇÚCAR ${ }^{1}$
}

\author{
Efficacy of the Herbicide Amicarbazone Applied on Straw or Soil for Weed Control in \\ Sugar Cane
}

\author{
TOLEDO, R.E.B. ${ }^{2}$, PERIM, L. ${ }^{3}$, NEGRISOLI, E. ${ }^{4}$, CORREA, M.R. ${ }^{5}$, CARBONARI, C.A. ${ }^{6}$, \\ ROSSI, C.V.S. ${ }^{7}$ e VELINI, E.D. ${ }^{8}$
}

\begin{abstract}
RESUMO - A eficácia dos herbicidas no sistema de cana-crua é diretamente influenciada pela quantidade e distribuição de palha na área, bem como pela ocorrência da primeira chuva superior a $20 \mathrm{~mm}$ posterior à aplicação, uma vez que a palha é capaz de interceptar o herbicida antes que este atinja o solo. Nesse contexto, a presente pesquisa teve como objetivo avaliar a eficácia e o comportamento do herbicida amicarbazone no controle de plantas daninhas no sistema de cana-crua. Para isso, foi realizado um experimento em condições controladas, considerando diferentes doses de amicarbazone $\left(D_{1}=1,05 \mathrm{~kg} \mathrm{ha}^{-1}\right.$, $\mathrm{D}_{2}=0,875 \mathrm{~kg} \mathrm{ha}^{-1}, \mathrm{D}_{3}=0,700 \mathrm{~kg} \mathrm{ha}^{-1}$ e $\mathrm{D}_{4}=0,525 \mathrm{~kg} \mathrm{ha}^{-1}$ ) e situações de aplicação desse herbicida, a saber: sobre $5 \mathrm{t}$ de palha ha ${ }^{-1}$, sobre o solo e coberto com $5 \mathrm{t}$ de palha ha ${ }^{-1}$, alem de pulverização sobre o solo sem cobertura de palha, resultando assim em 12 tratamentos. Aos 7, 14, 21 e 28 dias após a aplicação (DAA), foi avaliada a porcentagem de controle das seguintes espécies de plantas daninhas: Ipomoea grandifolia, Brachiaria decumbens, Merremia cissoides e Euphorbia heterophylla. Pelos resultados obtidos, pode-se constatar que, independentemente da espécie de planta daninha avaliada, os maiores indices de controle foram observados quando o amicarbazone foi aplicado sobre a palha, simulando-se em seguida precipitação correspondente a $30 \mathrm{~mm}$ de chuva, e nos tratamentos em que o herbicida foi aplicado diretamente no solo sem palha. Dessa forma, para as espécies I. grandifolia, $M$. cissoides e E. heterophylla, todos os tratamentos apresentaram excelente controle (nota $6-100 \%$ ) a partir dos 14 DAA; para B. decumbens, o controle total ocorreu aos 28 DAA. Portanto, pode-se concluir que o amicarbazone é uma excelente alternativa para o manejo dessas espécies de plantas daninhas em cana-crua.
\end{abstract}

Palavras-chave: herbicida, Convulvolaceae, cana-crua.

ABSTRACT - Herbicide efficacy in raw sugar cane is directly influenced by the amount and distribution of straw in the area, as well as by the occurrence of the first rainfall exceeding $20 \mathrm{~mm}$ after application, since straw is able to intercept the herbicide before it strikes the soil. Within this context, the present study aimed to evaluate the effectiveness and performance of the herbicide amicarbazone in weed control in raw cane. Thus, an experiment was conducted under controlled conditions considering different doses of amicarbazone $\left(D 1=1.05 \mathrm{~kg} \mathrm{ha}^{-1} ; D 2=0875 \mathrm{~kg} \mathrm{ha}^{-1} ; D 3=\right.$ $0700 \mathrm{~kg} \mathrm{ha}{ }^{-1}$ and D4 $\left.=0525 \mathrm{~kg} \mathrm{ha}^{-1}\right)$ and herbicide applications on 5 tonnes of straw ha-1 on the ground and covered with 5 tonnes of straw. ha ${ }^{-1}$; in addition to spraying on the ground without straw cover, resulting in 12 treatments. At 7, 14,21 and 28 days after application (DAA) the

1 Recebido para publicação em 6.6.2008 e na forma revisada em 5.6.2009.

2 Eng-ô-Agr ${ }^{0}$, Dr., Coordenador de Pesquisa e Desenvolvimento - Herbicidas, Arysta LifeScience, Rua Jundiaí, 50, 9aandar-Paraíso, 04001-904 São Paulo-SP, <roberto.toledo@arystalifescience.com>; ${ }^{3}$ Graduação do curso de Agronomia da FCA/Unesp, Botucatu-SP, <lperim@fca.unesp.br>; ${ }^{4}$ Eng - -Agr ${ }^{\circ}$, Dr., TechField-Nupam/FCA/Unesp, Botucatu-SP, <eduardo.negrisoli@gmail.com>; ${ }^{5}$ Engo-Agr ${ }^{0}$, Dr., TechField-Nupam/FCA/Unesp, Botucatu-SP, <marcelorcorrea@uol.com.br>; ${ }^{6}$ Engo-Agr ${ }^{\circ}$, MSc., FCA/Unesp, Botucatu-SP, <carbonari@fca.unesp.br>; ${ }^{7}$ Engo-Agr ${ }^{0}$, Dr., TechField-Nupam/FCA/Unesp, Botucatu-SP, <cavsragro@msn.com>; ${ }^{8}$ Professor, Dr., Dep. de Agricultura FCA/Unesp - Fazenda Experimental Lageado, Caixa Postal 237, 18603-970 Botucatu-SP, <velini@fca.unesp.br>

Planta Daninha, Viçosa-MG, v. 27, n. 2, p. 319-326, 2009 
percentage of control of the weed species Ipomoea grandifolia, Brachiaria decumbens, Merremia cissoides and Euphorbia heterophylla was evaluated. The results obtained showed that regardless of the type of weed assessed, the highest rates of control were observed when amicarbazone was applied on the straw, followed by simulated precipitation of $30 \mathrm{~mm}$ of rain and in treatments in which the herbicide was applied directly into the soil without straw. Thus, for the species I. grandifolia, M. cissoides and $\boldsymbol{E}$. heterophylla, all the treatments had excellent control (grade 6 to $100 \%$ ) after 14 DAA; for B. decumbens, complete control occurred at 28 DAA. Therefore, we can conclude that amicarbazone is an excellent alternative for the management of these species of weeds in raw cane.

Keywords: herbicide, convolvulaceae, sugar cane

\section{INTRODUÇÃO}

Na colheita de cana-crua, dependendo da variedade utilizada, são deixadas sobre o solo de 5 a 20 toneladas de palha por hectare (Velini $\&$ Negrisoli, 2000). A quantidade de palha também é função direta das características da variedade, como facilidade de despalha do colmo, hábito de crescimento da touceira, uniformidade em altura e tamanho dos ponteiros, produtividade e desenvolvimento da cana.

A palha é apenas uma das barreiras para o uso de herbicidas com ação exclusiva ou preferencial no solo. O acréscimo do teor superficial de matéria orgânica no solo, menos evidente em cana-crua do que em plantio direto, em razão da movimentação mínima no momento da colheita mecanizada associada ao preparo e sulcamento quando da reimplantação da cultura, propicia a adsorção dos herbicidas, limitando a sua eficiência (Negrisoli et al., 2005). Algumas plantas daninhas, como Ipomoea grandifolia e Euphorbia heterophylla, não têm sua germinação inibida pelas quantidades de palha de cana-de-açúcar que normalmente são encontradas em campo (Martins et al., 1999; Velini \& Negrisoli, 2000; Negrisoli et al., 2007a).

A grande redução inicial na emergência das principais espécies de plantas daninhas na cultura da cana, após a colheita sem queima, onde a quantidade de palha sobre o solo é máxima, pode levar a uma falsa impressão de que em áreas nessas condições o controle de plantas daninhas poderá ser suprimido apenas pela presença da palha (Velini \& Negrisoli, 2000) ou com a utilização de subdosagens de herbicidas.

Lamoreaux et al. (1993) ressaltam que a lavagem de herbicidas da palha para o solo é dependente da capacidade desses resíduos em cobrir o solo e reter os herbicidas; da solubilidade do produto aplicado; e do período em que a área permanece sem chuva após a aplicação do produto. A partir do momento em que esses herbicidas atingem o solo, apresentam maior distribuição e persistência, devido aos canais formados pelos restos vegetais (formando vias preferenciais de escoamento) ou por organismos do solo (minhocas) e, principalmente, pela amenização dos processos de degradação (Jones Jr. et al., 1990; Sorenson et al., 1991).

Além disso, da mesma forma que esses resíduos de colheita deixados sobre o solo são uma barreira para as plantas daninhas atingirem a superficie, tendo acesso à luz solar, também são uma barreira para que os herbicidas aplicados sobre a palha possam atingir o solo e atuar normalmente. Em culturas em que o controle deve ser feito por longos períodos, em razão dos espaçamentos amplos, essa limitação é bastante importante. Em áreas de cana-crua o espaçamento entre linhas da cana foi ampliado para 1,4 a $1,5 \mathrm{~m}$, dificultando o fechamento da área, sobretudo em solos com menor fertilidade.

Correia \& Durigan (2004) estudaram os efeitos da cobertura do solo, com 0, 5, 10 e $15 \mathrm{t} \mathrm{ha}^{-1}$ de palha de cana-de-açúcar da variedade SP 79 2233, sobre a emergência de seis espécies de plantas daninhas (Brachiaria decumbens, Digitaria horizontalis, Sida spinosa, Ipomoea grandifolia, Ipomoea hederifolia e Ipomoea quamoclit), e constataram que a presença de palha sobre a superficie do solo inibiu a emergência de plântulas das espécies $B$. decumbens e $S$. spinosa, sendo o mesmo observado para $D$. horizontalis, quando submetidas 
a 10 e 15 t ha $^{-1}$ de palha. No entanto, para I. grandifolia e I. hederifolia o número de plantas emersas não diferiu entre as quantidades de palha. Por sua vez, a presença da cobertura morta incrementou a emergência de plântulas de I. quamoclit. Após a remoção da palha, os autores não verificaram fluxos expressivos na emergência de plântulas das espécies estudadas.

Segundo Rodrigues \& Almeida (2005), o mecanismo de ação principal do amicarbazone é pela inibição da fotossintese das plantas daninhas, atuando na Reação de Hill (Fotossistema II), inibindo o transporte de elétrons e paralisando a fixação de $\mathrm{CO}_{2}$ e a produção de ATP e $\mathrm{NADPH}_{2}$, os quais são elementos essenciais para o crescimento das plantas. A morte de plantas, entretanto, pode ocorrer devido a outros processos, como a peroxidação de lipídeos e proteínas, promovendo a destruição das membranas e perda de clorofila. O amicarbazone é um herbicida recomendado para aplicações em pré e pósemergência inicial para o controle de um amplo espectro de plantas daninhas que competem com a lavoura canavieira nas doses de 1,50 a 2,0 $\mathrm{kg} \mathrm{ha}^{-1}$. Quando aplicado em préemergência, o amicarbazone é absorvido pelo sistema radicular e translocado via xilema, pelo fluxo de transpiração.
Nesse contexto, este trabalho teve como objetivo avaliar a eficácia e o comportamento do herbicida amicarbazone no controle de plantas daninhas no sistema de cana-crua.

\section{MATERIAL E MÉTODOS}

A presente pesquisa foi realizada em condições controladas de casa de vegetação no Núcleo de Pesquisas Avançadas em Matologia (NUPAM), pertencente ao Departamento de Produção Vegetal da Faculdade de Ciências Agronômicas/UNESP - Botucatu-SP.

O solo utilizado como substrato foi inicialmente seco à sombra por um período de 48 horas. Depois disso, foi peneirado em peneira com malha de 200 mesh, sendo submetido às análises químicas e granulométricas (Tabelas 1 e 2). Com base nesses resultados, o solo foi adubado para propiciar uma melhor germinação das plantas daninhas e, em segui$\mathrm{da}$, acondicionado em vasos de $2 \mathrm{~L}$ de capacidade, procedendo-se à semeadura com as espécies Ipomoea grandifolia (IAQGR), Brachiaria decumbens (BRADC), Merremia cissoides (MRRCI) e Euphorbia heterophylla (EPHHL). O trabalho foi conduzido em delineamento inteiramente casualizado, com quatro repetições, incluindo-se testemunhas com e sem a palha da cana-de-açúcar, para avaliação do controle

Tabela 1 - Análise química da amostra de solo utilizado nos vasos - experimento de eficácia do herbicida amicarbazone em diferentes posicionamentos sobre a palha de cana-de-açúcar. Botucatu, 2007

\begin{tabular}{|c|c|c|c|c|c|c|c|c|c|c|c|}
\hline \multirow{2}{*}{ Solo } & $\mathrm{PH}$ & M.O. & P res. & $\mathrm{K}^{+}$ & $\mathrm{Ca}^{+2}$ & $\mathrm{Mg}^{+2}$ & $\mathrm{H}^{+}+\mathrm{Al}^{+3}$ & $\mathrm{SB}$ & $\mathrm{T}$ & $\mathrm{V}$ \\
\cline { 2 - 11 } & $\left(\mathrm{CaCl}_{2)}\right.$ & $\left(\mathrm{g} \mathrm{dm}^{-3}\right)$ & $\left(\mathrm{mg} \mathrm{dm}^{-3}\right)$ & \multicolumn{7}{|c|}{$\left(\mathrm{mmol}_{\mathrm{c}} \mathrm{dm}^{-3}\right)$} & \multicolumn{3}{|c|}{$(\%)$} \\
\hline \multirow{2}{*}{$\mathrm{LVd}$} & 4,3 & 19 & 1 & 0,6 & 10 & 4 & 58 & 14,6 & 73 & 21 \\
\hline
\end{tabular}

Departamento de Solos - FCA/UNESP - Botucatu.

Tabela 2 -Análise granulométrica do solo utilizado no experimento de eficácia do herbicida amicarbazone em diferentes posicionamentos sobre a palha de cana-de-açúcar. Botucatu, 2007

\begin{tabular}{|c|c|c|c|c|c|c|c|}
\hline \multirow{3}{*}{$\begin{array}{c}\text { Granulometria } \\
(\%)\end{array}$} & \multicolumn{7}{|c|}{ Classe de solo } \\
\hline & \multirow{2}{*}{ Argila } & \multirow{2}{*}{ Limo } & \multicolumn{4}{|c|}{ Areia } & \multirow{2}{*}{ Classe textural } \\
\hline & & & Fina & Média & Grossa & Total & \\
\hline LVd & 20 & 4 & 22,9 & 35,7 & 17,4 & 76 & Média \\
\hline
\end{tabular}

Departamento de Solos - FCA/UNESP - Botucatu. 
proporcionado pelo amicarbazone. A umidade do solo, medida gravimetricamente, foi mantida durante todo o ensaio em $65 \%$ da capacidade de campo.

A palha de cana-de-açúcar utilizada no experimento foi a variedade SP 80-1816, na quantidade de $5 \mathrm{t} \mathrm{ha}^{-1}$; essa quantidade foi selecionada em razão das informações disponíveis na literatura e de estudos anteriormente realizados, indicando ser esta quantidade uma das condições mais críticas em termos de manejo de plantas daninhas em cana-crua, uma vez que é insuficiente para controlar adequadamente a emergência das plantas daninhas, mas o suficiente para reter quase que completamente a quantidade de calda aplicada (Velini $\&$ Negrisoli, 2000).

A pulverização do herbicida e a simulação de chuva foram realizadas através de um equipamento instalado em laboratório do NuPAM, o qual é constituído de uma estrutura metálica com $3 \mathrm{~m}$ de altura por $2 \mathrm{~m}$ de largura, que permite acoplamento de um "carrinho" suspenso a 2,5 m de altura. A esse carrinho encontram-se acopladas duas barras de pulverização, uma responsável pelo sistema de simulação de chuva e a outra pelo sistema de pulverização de defensivos agrícolas, as quais se deslocam por uma área útil de $6 \mathrm{~m}^{2}$ no sentido do comprimento do equipamento. $\mathrm{O}$ tracionamento de ambas as barras é realizado por meio de correntes e engrenagens, com auxilio de um motor elétrico, cujo ajuste é dado por um modulador de frequência, permitindo a obtenção de velocidade constante previamente determinada. $\mathrm{O}$ sistema de pulverização tem funcionamento independente do sistema de simulação de chuva, apesar de ambos compartilharem o mesmo equipamento e funções de controle.

A simulação da chuva foi realizada utilizando-se uma bomba hidráulica de pressão constante e acionamento automático, a qual bombeia água armazenada de um reservatório, com capacidade para $1.000 \mathrm{~L}$, até a barra e pontas de pulverização responsáveis pela formação de gotas de chuva. Essa barra, situada a 1,45 m de altura em relação à superficie das unidades experimentais, é constituída por três bicos de pulverização TK-SS-20 de alta vazão, espaçados de $0,5 \mathrm{~m}$ e posicionados de forma a propiciar maior uniformidade de precipitação na área aplicada. Esse sistema foi operado com velocidade de deslocamento de $0,187 \mathrm{~km} \mathrm{~h}^{-1}$, o que correspondeu a 2,5 Hertz no modulador de frequência e pressão de trabalho de 0,81 $\mathrm{kgf} \mathrm{s}^{-2}$. Essas especificações proporcionaram a produção de gotas artificiais de chuva com diâmetro mediano volumétrico (DMV) de 1.140 micras, conforme informações do fabricante da ponta de pulverização (Spraying Systems Co.), e cada lâmina aplicada correspondeu a aproximadamente $2,5 \mathrm{~mm}$ de chuva simulada.

A barra de pulverização era constituída por quatro pontas de pulverização XR 11002 VS, espaçadas entre si em $0,5 \mathrm{~m}$ e posicionadas a $0,5 \mathrm{~m}$ de altura em relação à superfície das unidades experimentais. Para as pulverizações do herbicida, o sistema foi operado com velocidade de deslocamento de $3,6 \mathrm{~km} \mathrm{~h}^{-1}$, o que correspondeu a 45,0 Hertz no modulador de frequência, com consumo de calda correspondente a $200 \mathrm{~L} \mathrm{ha}^{-1}$. O equipamento foi operado sob pressão constante de 1,5 bar, pressurizado por ar comprimido. As doses de amicarbazone aplicadas foram D1 $=1,05 \mathrm{~kg}$ amicarbazone ha-1; $\mathrm{D} 2=0,875 \mathrm{~kg}$ amicarbazone ha- $\mathrm{ha}^{-1} \mathrm{D} 3=$ $0,70 \mathrm{~kg}$ amicarbazone ha-1; e $\mathrm{D} 4=$ $0,525 \mathrm{~kg}$ amicarbazone ha-1.

Na Tabela 3 são dispostos os tratamentos utilizados no experimento conduzido em vasos com diferentes posicionamentos do herbicida amicarbazone.

O controle das espécies de plantas daninhas foi determinado por meio de uma escala visual e percentual de notas, variando de zero a 100. Nesse caso, zero consistia em nenhum controle e 100 era a porcentagem de controle total das espécies daninhas. As avaliações foram realizadas até o desaparecimento ou a

Tabela 3 - Escala de notas da ALAM utilizada para avaliação da eficácia de controle de plantas daninhas

\begin{tabular}{|c|l|}
\hline Porcentagem (\%) & Grau de controle \\
\hline $0-40$ & Nenhum a pobre (1) \\
\hline $41-60$ & Regular (2) \\
\hline $61-70$ & Suficiente (3) \\
\hline $71-80$ & Bom (4) \\
\hline $81-90$ & Muito Bom (5) \\
\hline $91-100$ & Excelente (6) \\
\hline
\end{tabular}


estabilização dos sintomas de fitointoxicação (SBCPD, 1995), sendo também correlacionadas com a escala de notas da Asociación LatinoAmericana de Malezas (ALAM, 1974), apresentada na Tabela 4, a qual varia de 1 (nenhum a pobre) a 6 (excelente controle). Os dados foram comparados estatisticamente pelo teste de Tukey a 5\% de probabilidade.

\section{RESULTADOS E DISCUSSÃO}

De acordo com os dados contidos na Tabela 5, aos sete dias após a aplicação do herbicida amicarbazone (DAA) foi observada baixa eficiência de controle dos diferentes tratamentos propostos sobre todas as espécies de plantas daninhas. As espécies convolvuláceas apresentaram maior sensibilidade aos tratamentos, com médias variando de 8,5 a $17,5 \%$ de controle. Para $B$. decumbens, nesse primeiro período de avaliação as plantas emergidas sofreram baixa interferência dos tratamentos, com médias de controle de até $5 \%$. No caso de E. heterophylla, com exceção do tratamento com aplicação de amicarbazone na dose de $0,75 \mathrm{~kg}$ p.c. ha ${ }^{-1}$ seguido de precipitação, todos os tratamentos não apresentaram efeito sobre essa espécie de planta daninha. Contudo, mesmo com os tratamentos apresentando baixa eficácia de controle, foram observados sintomas iniciais de intoxicação nas plantas daninhas, caracterizados por cloroses foliares.

Já aos 14 DAA (Tabela 6), verificou-se que todos os tratamentos apresentaram excelente nivel de controle para todas as espécies avaliadas, com médias superiores, em sua maioria, a 97\% (nota 6 em referência à escala ALAM). Nesse caso, com exceção da espécie $B$. decumbens, as demais espécies de plantas daninhas foram eficientemente controladas pelos tratamentos propostos. A eficácia de controle sobre esta espécie em questão variou bastante entre os tratamentos. Em todas as doses testadas, quando o herbicida foi aplicado sobre a palha, em simulação a uma aplicação comercial convencional, e a precipitação ocorreu após 24 horas (PAC), os resultados médios foram estatisticamente inferiores, em comparação à aplicação de amicarbazone sobre o solo desnudo (AC) ou quando aplicado anteriormente à deposição de palha na superficie do solo (APC).

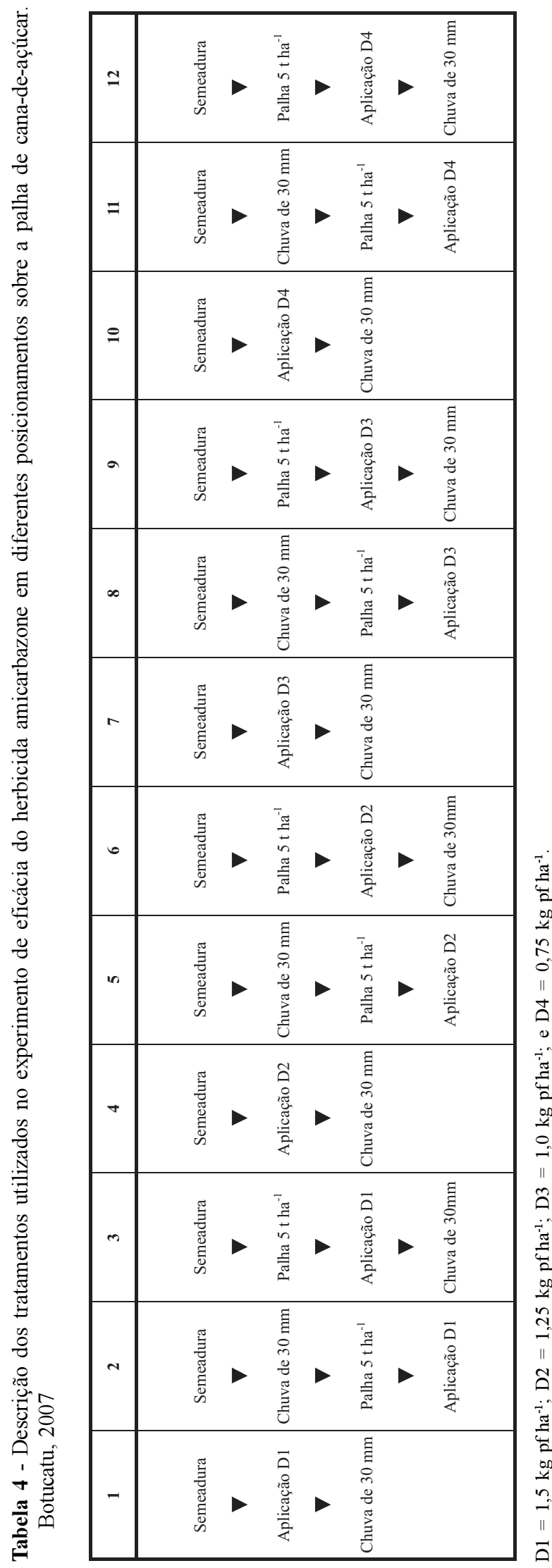

Planta Daninha, Viçosa-MG, v. 27, n. 2, p. 319-326, 2009 
Tabela 5 - Porcentagem média e notas segundo a escala da ALAM de controle de I. grandifolia, B. decumbens, $M$. cissoides e E. heterophylla pelo herbicida amicarbazone aos 7 DAA. Botucatu, 2007

\begin{tabular}{|c|c|c|c|c|c|}
\hline \multirow{2}{*}{ Tratamento } & \multicolumn{5}{|c|}{$7 \mathrm{DAA}$} \\
\hline & \multicolumn{2}{|l|}{ IAQGR } & BRADC & MRRCI & EPHHL \\
\hline 1. D1 (AC) & $12,50 \mathrm{abcd}$ & $1^{1 /}$ & $1,25 \mathrm{ab}$ & $10,00 \mathrm{a}$ & $0,00 \mathrm{~b} \quad 1$ \\
\hline 2. D1 (APC) & $8,50 \mathrm{~d}$ & 1 & $0,00 \mathrm{~b}$ & $8,75 \mathrm{a}$ & $0,00 \mathrm{~b} \quad 1$ \\
\hline 3. D1 (PAC) & $11,50 \mathrm{bcd}$ & 1 & $0,00 \mathrm{~b}$ & $7,50 \mathrm{a}$ & $0,00 \mathrm{~b} \quad 1$ \\
\hline 4. D2 (AC) & $10,00 \mathrm{~cd}$ & 1 & $0,00 \mathrm{~b}$ & $10,00 \mathrm{a}$ & $0,00 \mathrm{~b} \quad 1$ \\
\hline 5. D2 (APC) & $17,50 \mathrm{a}$ & 1 & $3,75 \mathrm{ab}$ & $13,75 \mathrm{a}$ & $0,00 \mathrm{~b} \quad 1$ \\
\hline 6. D2 (PAC) & $12,50 \mathrm{abcd}$ & 1 & $2,50 \mathrm{ab}$ & $11,25 \mathrm{a}$ & $0,00 \mathrm{~b} \quad 1$ \\
\hline 7. D3 (AC) & $8,50 \mathrm{~d}$ & 1 & $1,25 \mathrm{ab}$ & $11,25 \mathrm{a}$ & $0,00 \mathrm{~b} \quad 1$ \\
\hline 8. D3 (APC) & $15,00 \mathrm{abc}$ & 1 & $5,00 \mathrm{a}$ & $10,00 \mathrm{a}$ & $0,00 \mathrm{~b} \quad 1$ \\
\hline 9. D3 (PAC) & $12,25 \mathrm{bcd}$ & 1 & $1,25 \mathrm{ab}$ & $10,00 \mathrm{a}$ & $0,00 \mathrm{~b} \quad 1$ \\
\hline 10.D4 (AC) & $13,00 \mathrm{abcd}$ & 1 & $0,00 \mathrm{~b}$ & $12,50 \mathrm{a}$ & $3,75 \mathrm{a} \quad 1$ \\
\hline 11.D4 (APC) & $16,25 \mathrm{ab}$ & 1 & $3,75 a b$ & $15,00 \mathrm{a}$ & $0,00 \mathrm{~b} \quad 1$ \\
\hline 12.D4 (PAC) & $16,25 \mathrm{ab}$ & 1 & $3,75 \mathrm{ab}$ & $10,00 \mathrm{a}$ & $0,00 \mathrm{~b} \quad 1$ \\
\hline $\begin{array}{l}\mathrm{F} \\
\mathrm{DMS} \\
\mathrm{CV} \%\end{array}$ & $\begin{array}{r}6,14 \\
5,90 \\
18,74\end{array}$ & & $\begin{array}{r}3,42 \\
4,82 \\
104,23\end{array}$ & $\begin{array}{c}1,87^{\mathrm{ni}} \\
7,92 \\
30,08\end{array}$ & $\begin{array}{r}2,45 \\
3,41 \\
442,22\end{array}$ \\
\hline
\end{tabular}

1/ Notas segundo a escala da ALAM.

Obs.: médias seguidas de mesma letra, nas colunas, não diferem entre si pelo teste de Tukey a $5 \%$ de probabilidade.

** Significativo pelo teste $\mathrm{F}$ a $1 \%$ de probabilidade; * significativo pelo teste $\mathrm{F}$ a $5 \%$ de probabilidade; ${ }^{\text {ns }}$ não-significativo.

IAQGR: Ipomoea grandifolia; BRADC: Brachiaria decumbens; MRRCI: Merremia cissoides; EPHHL: Euphorbia heterophylla.

D1 $=1,05 \mathrm{~kg}$ i.a.ha $a^{-1} ;$ D2 $=0,875 \mathrm{~kg}$ i.a.ha $a^{-1} ;$ D3 = 0,70 kg i.a.ha ${ }^{-1}$; D4 $=0,525 \mathrm{~kg}$ i.a.ha ${ }^{-1}$.

$\mathrm{A}=$ aplicação de amicarbazone; $\mathrm{P}=$ cobertura com $5 \mathrm{t} \mathrm{ha}^{-1}$ de palha de cana-de-açúcar; C = chuva de $30 \mathrm{~mm} 24$ horas após aplicação.

A mesma tendência também foi observada na avaliação ocorrida aos 21 DAA (Tabela 7). Ocorreu pequena variação entre as médias de controle dos tratamentos para I. grandifolia, $M$. cissoides e E. heterophylla, com controle total dessas espécies para a maioria dos tratamentos, independentemente da dosagem de amicarbazone utilizada e da presença de palha em cobertura. Para $B$. decumbens, a variação das médias de controle foi mais discrepante: entre 97 e 100\%. Apesar disso, não ocorreu diferença significativa entre os tratamentos testados para nenhuma das espécies de plantas daninhas avaliadas.

$\mathrm{Na}$ avaliação ocorrida aos 28 DAA, verificou-se que os tratamentos proporcionaram controle total das quatro espécies de plantas daninhas avaliadas (Tabela 8). Esses resultados
Tabela 6 - Porcentagem média e notas segundo a escala da ALAM (1974) de controle de I. grandifolia, B. decumbens, $M$. cissoides e E. heterophylla pelo herbicida amicarbazone aos 14 DAA. Botucatu, 2007

\begin{tabular}{|c|c|c|c|c|c|}
\hline \multirow{2}{*}{ Tratamento } & \multicolumn{5}{|c|}{14 DAA } \\
\hline & IAQGR & BRADC & & MRRCI & EPHHL \\
\hline 1. D1 (AC) & $98,00 \mathrm{a} \quad 6^{1 /}$ & $82,50 \mathrm{abc}$ & 5 & 98,50 a 6 & 99,25 a 6 \\
\hline 2. D1 (APC) & 99,25 a 6 & $83,75 a b c$ & 5 & 100,00 a 6 & 99,75 a 6 \\
\hline 3. D1 (PAC) & 99,75 a 6 & $75,00 \mathrm{c}$ & 4 & 99,25 a 6 & 98,5 o a 6 \\
\hline 4. D2 (AC) & $99,00 \mathrm{a} \quad 6$ & $96,25 \mathrm{a}$ & 6 & 99,25 a 6 & 99,25 a 6 \\
\hline 5. D2 (APC) & 99,75 a 6 & $94,50 \mathrm{a}$ & 6 & 99,75 a 6 & 99,25 a 6 \\
\hline 6. D2 (PAC) & 99,25 a 6 & $77,75 \mathrm{bc}$ & 4 & 99,75 a 6 & $100,00 \mathrm{a} \quad 6$ \\
\hline 7. D3 (AC) & 98,00 a 6 & $92,50 \mathrm{a}$ & 5 & 99,00 a 6 & 99,75 a 6 \\
\hline 8. D3 (APC) & 99,75 a 6 & $90,75 \mathrm{ab}$ & 5 & 99,50 a 6 & 99,75 a 6 \\
\hline 9. D3 (PAC) & 99,25 a 6 & $83,25 \mathrm{abc}$ & 4 & 99,75 a $\quad 6$ & 99,25 a 6 \\
\hline 10.D4 (AC) & 99,75 a 6 & $77,50 \mathrm{bc}$ & 6 & 99,25 a 6 & 97,50 a 6 \\
\hline 11.D4 (APC) & 100,00 a 6 & $98,50 \mathrm{abc}$ & 6 & 99,75 a 6 & 99,25 a 6 \\
\hline 12.D4 (PAC) & 100,00 a 6 & $77,25 \mathrm{bc}$ & 4 & 99,75 a 6 & 99,75 a 6 \\
\hline $\begin{array}{l}\text { DMS } \\
\mathrm{CV} \%\end{array}$ & $\begin{array}{l}1,55^{\mathrm{ns}} \\
3,00 \\
1,23\end{array}$ & $\begin{array}{r}6,68 \\
13,93 \\
6,66\end{array}$ & & $\begin{array}{l}1,44^{1 \mathrm{~s}} \\
1,74 \\
0,71\end{array}$ & $\begin{array}{l}1,28^{\text {ns }} \\
3,13 \\
1,28\end{array}$ \\
\hline
\end{tabular}

${ }^{1 /}$ Notas segundo a escala da ALAM.

Obs.: médias seguidas de mesma letra, nas colunas, não diferem entre si pelo teste de Tukey a $5 \%$ de probabilidade.

** Significativo pelo teste $\mathrm{F}$ a $1 \%$ de probabilidade; * significativo pelo teste F a $5 \%$ de probabilidade; ${ }^{\text {ns }}$ não-significativo.

IAQGR: Ipomoea grandifolia; BRADC: Brachiaria decumbens; MRRCI: Merremia cissoides; EPHHL: Euphorbia heterophylla.

D1 $=1,05 \mathrm{~kg}$ i.a.ha-1; D2 =0,875 kg i.a.ha ${ }^{-1} ;$ D3 = 0,70 kg i.a.ha-1; D4 $=0,525 \mathrm{~kg}$ i.a.ha ${ }^{-1}$.

$\mathrm{A}=$ aplicação de amicarbazone; $\mathrm{P}=$ cobertura com $5 \mathrm{t} \mathrm{ha}^{-1}$ de palha de cana-de-açúcar; $\mathrm{C}=$ chuva de $30 \mathrm{~mm} 24$ horas após aplicação.

indicaram que, independentemente da dosagem de amicarbazone utilizada e do posicionamento do produto durante a aplicação, os resultados foram considerados satisfatórios para essas espécies invasoras da cultura da canade-açúcar nesse período final de avaliações.

Negrisoli et al. (2007b), trabalhando com tebuthiuron, também observaram que a aplicação sobre a palha de cana-de-açúcar resultou em excelente controle da espécie I. grandifolia, com médias de $100 \%$ nesse mesmo periodo, quando ocorreu uma precipitação de $20 \mathrm{~mm}$ 24 horas após a pulverização. Esse resultado também foi observado por Negrisoli et al. (2009) trabalhando com o herbicida oxyfluorfen.

Independentemente da planta daninha estudada, todos os tratamentos apresentaram as maiores porcentagens médias de controle 
Tabela 7 - Porcentagem média e notas segundo a escala daALAM de controle de I. grandifolia, B. decumbens, $M$. cissoides e E. heterophylla pelo herbicida amicarbazone aos 21 DAA. Botucatu, 2007

\begin{tabular}{|c|c|c|c|c|}
\hline \multirow{2}{*}{ Tratamento } & \multicolumn{4}{|c|}{$21 \mathrm{DAA}$} \\
\hline & IAQGR & BRADC & MRRCI & EPHHL \\
\hline 1. D1 (AC) & $100,00 \mathrm{a} \quad 6^{-1 /}$ & $99,75 \mathrm{a}$ & $100,00 \mathrm{a} \quad 6$ & 99,75 a 6 \\
\hline 2. D1 (APC) & 99,75 a 6 & $99,75 \mathrm{a}$ & 99,75 a 6 & 100,00 a 6 \\
\hline 3. D1 (PAC) & 99,75 a 6 & $99,25 \mathrm{a}$ & $100,00 \mathrm{a} \quad 6$ & 100,00 a 6 \\
\hline 4. D2 (AC) & 100,00 a 6 & $100,00 \mathrm{a}$ & 100,00 a 6 & 100,00 a 6 \\
\hline 5. D2 (APC) & 99,75 a 6 & $99,50 \mathrm{a}$ & 100,00 a 6 & 100,00 a 6 \\
\hline 6. D2 (PAC) & 100,00 a 6 & $98,50 \mathrm{a}$ & $100,00 \mathrm{a} \quad 6$ & 100,00 a 6 \\
\hline 7. D3 (AC) & 100,00 a 6 & $99,25 \mathrm{a}$ & $100,00 \mathrm{a} \quad 6$ & 99,85 a 6 \\
\hline 8. D3 (APC) & 100,00 a 6 & $99,25 \mathrm{a}$ & $100,00 \mathrm{a} \quad 6$ & 100,00 a 6 \\
\hline 9. D3 (PAC) & 100,00 a 6 & $97,00 \mathrm{a}$ & 100,00 a 6 & 100,00 a 6 \\
\hline 10.D4 (AC) & 100,00 a 6 & $98,70 \mathrm{a}$ & $100,00 \mathrm{a} \quad 6$ & 98,25 a 6 \\
\hline 11.D4 (APC) & 100,00 a 6 & $98,50 \mathrm{a}$ & $100,00 \mathrm{a} \quad 6$ & 100,00 a 6 \\
\hline 12.D4 (PAC) & 100,00 a 6 & $97,25 \mathrm{a}$ & 99,50 a 6 & 100,00 a 6 \\
\hline $\begin{array}{l}\mathrm{F} \\
\mathrm{DMS} \\
\mathrm{CV} \%\end{array}$ & $\begin{array}{l}0,82^{\mathrm{ns}} \\
0,62 \\
0,25\end{array}$ & $\begin{array}{l}0,82^{\mathrm{ns}} \\
5,46 \\
2,24\end{array}$ & $\begin{array}{l}-1,99^{n \mathrm{~ns}} \\
0,54 \\
0,22\end{array}$ & $\begin{array}{l}2,07^{n s} \\
1,79 \\
0,73\end{array}$ \\
\hline
\end{tabular}

${ }^{1 /}$ Notas segundo a escala da ALAM.

Obs.: médias seguidas de mesma letra, nas colunas, não diferem entre si pelo teste de Tukey a $5 \%$ de probabilidade.

** Significativo pelo teste $\mathrm{F}$ a $1 \%$ de probabilidade; * significativo pelo teste $\mathrm{F}$ a $5 \%$ de probabilidade; ${ }^{\text {ns }}$ não-significativo.

IAQGR: Ipomoea grandifolia; BRADC: Brachiaria decumbens; MRRCI: Merremia cissoides; EPHHL: Euphorbia heterophylla.

$\mathrm{D} 1=1,05 \mathrm{~kg}$ i.a.ha-1; D2 $=0,875 \mathrm{~kg}$ i.a.ha ${ }^{-1} ; \mathrm{D} 3=0,70 \mathrm{~kg}$ i.a.ha-1; D4 $=0,525 \mathrm{~kg}$ i.a.ha-1 .

$\mathrm{A}=$ aplicação de amicarbazone; $\mathrm{P}=$ cobertura com $5 \mathrm{t} \mathrm{ha}^{-1}$ de palha de cana-de-açúcar; $\mathrm{C}$ = chuva de $30 \mathrm{~mm} 24$ horas após aplicação.

aos 28 DAA, provavelmente pelo fato de que o amicarbazone atingiu o solo seja através da aplicação direta ou através da lixiviação do herbicida, promovida pela simulação da chuva após sua aplicação.

Dessa forma, pode-se concluir que a espécie I. grandifolia mostrou-se mais sensivel ao amicarbazone, demonstrando menor tolerância aos tratamentos com o herbicida nas diferentes épocas de avaliação. Para $B$. decumbens, as porcentagens de controle foram altas nos tratamentos em que o herbicida atingiu o solo, independentemente se aplicado diretamente sobre este ou lixiviado pela simulação de chuva após a aplicação do herbicida. O amicarbazone é absorvido pelo sistema radicular e translocado via xilema. No entanto, o predomínio da absorção foliar ocorre
Tabela 8 - Porcentagem média e notas segundo a escala daALAM de controle de I. grandifolia, B. decumbens, $M$. cissoides e E. heterophylla pelo herbicida amicarbazone aos 28 DAA. Botucatu, 2007

\begin{tabular}{|c|c|c|c|c|}
\hline \multirow{2}{*}{ Tratamento } & \multicolumn{4}{|c|}{$28 \mathrm{DAA}$} \\
\hline & IAQGR & BRADC & MRRCI & EPHHL \\
\hline 1. D1 (AC) & $100,00 \mathrm{a} \quad 6^{-1 /}$ & 100,00 a 6 & 100,00 a 6 & 100,00 a 6 \\
\hline 2. D1 (APC) & 100,00 a 6 & $100,00 \mathrm{a} \quad 6$ & 100,00 a 6 & 100,00 a 6 \\
\hline 3. D1 (PAC) & 100,00 a 6 & $100,00 \mathrm{a} \quad 6$ & 100,00 a 6 & $100,00 \mathrm{a} \quad 6$ \\
\hline 4. D2 (AC) & 100,00 a 6 & $100,00 \mathrm{a} \quad 6$ & 100,00 a 6 & $100,00 \mathrm{a} \quad 6$ \\
\hline 5. D2 (APC) & 100,00 a 6 & $100,00 \mathrm{a} \quad 6$ & $100,00 \mathrm{a} \quad 6$ & 100,00 a 6 \\
\hline 6. D2 (PAC) & $100,00 \mathrm{a} \quad 6$ & $100,00 \mathrm{a} \quad 6$ & $100,00 \mathrm{a} \quad 6$ & 100,00 a 6 \\
\hline 7. D3 (AC) & $100,00 \mathrm{a} \quad 6$ & $100,00 \mathrm{a} \quad 6$ & $100,00 \mathrm{a} \quad 6$ & 100,00 a 6 \\
\hline 8. D3 (APC) & 100,00 a 6 & $100,00 \mathrm{a} \quad 6$ & $100,00 \mathrm{a} \quad 6$ & $100,00 \mathrm{a} \quad 6$ \\
\hline 9. D3 (PAC) & 100,00 a 6 & $100,00 \mathrm{a} \quad 6$ & $100,00 \mathrm{a} \quad 6$ & $100,00 \mathrm{a} \quad 6$ \\
\hline 10.D4 (AC) & 100,00 a 6 & $100,00 \mathrm{a} \quad 6$ & 100,00 a 6 & 100,00 a 6 \\
\hline 11.D4 (APC) & 100,00 a 6 & $100,00 \mathrm{a} \quad 6$ & 100,00 a 6 & $100,00 \mathrm{a} \quad 6$ \\
\hline 12.D4 (PAC) & 100,00 a 6 & $100,00 \mathrm{a} \quad 6$ & $100,00 \mathrm{a} \quad 6$ & 100,00 a 6 \\
\hline $\begin{array}{l}\text { F } \\
\text { DMS } \\
\text { CV \% }\end{array}$ & $\begin{array}{l}- \\
-\end{array}$ & $\begin{array}{l}- \\
-\end{array}$ & $\begin{array}{l}- \\
-\end{array}$ & $\begin{array}{l}- \\
-\end{array}$ \\
\hline
\end{tabular}

${ }^{1 /}$ Notas segundo a escala da ALAM.

Obs.: médias seguidas de mesma letra, nas colunas, não diferem entre si pelo teste de Tukey a $5 \%$ de probabilidade.

** Significativo pelo teste $\mathrm{F}$ a $1 \%$ de probabilidade; * significativo pelo teste $\mathrm{F}$ a $5 \%$ de probabilidade; ns não-significativo.

IAQGR: Ipomoea grandifolia; BRADC: Brachiaria decumbens; MRRCI: Merremia cissoides; EPHHL: Euphorbia heterophylla.

D1 $=1,05 \mathrm{~kg}$ i.a.ha ${ }^{-1} ; \mathrm{D} 2=0,875 \mathrm{~kg}$ i.a.ha ${ }^{-1} ; \mathrm{D} 3=0,70 \mathrm{~kg}$ i.a.ha ${ }^{-1}$; D4 $=0,525 \mathrm{~kg}$ i.a.ha ${ }^{-1}$.

$\mathrm{A}=$ aplicação de amicarbazone; $\mathrm{P}=$ cobertura com $5 \mathrm{tha}^{-1}$ de palha de cana-de-açúcar; $\mathrm{C}=$ chuva de $30 \mathrm{~mm} 24$ horas após aplicação.

quando ele é aplicado em pós-emergência, promovendo sua ação de contato. Os resultados observados neste estudo estão de acordo com os de Costa (2001), o qual relata que, para exercerem sua atividade sobre as plantas infestantes, os herbicidas residuais precisam ser introduzidos diretamente no solo, o que é proporcionado pelo carreamento através da água de chuva, em função da solubilidade do produto.

Pelos resultados obtidos, pode-se concluir que o herbicida Dinamic (amicarbazone) apresentou excelente eficácia no controle de corda-de-viola (Ipomoea grandifolia e Merremia cissoides), leiteiro (Euphorbia heterophylla) e capim-braquiária (Brachiaria decumbens), independentemente da dose utilizada $\left(0,75\right.$ a $\left.1,50 \mathrm{~kg} \mathrm{ha}^{-1}\right)$ e do posicionamento na palha ou no solo, demonstrando 
assim ser uma excelente alternativa para o controle de plantas daninhas em cana-deaçúcar crua ou queimada.

\section{LITERATURA CITADA}

ASOCIACIÓN LATINOAMERICANA DE MALEZAS ALAM. Recomendaciones sobre unificación de los sistemas de evaluación en ensayos de control de malezas. ALAM, v. 1, n. 1, p. $35-38,1974$

CORREIA, N. M.; DURIGAN, J. C. Emergência de plantas daninhas em solo coberto com palha de cana-de-açúcar.

Planta Daninha, v. 22, n. 1, p. 11-17, 2004.

JONES JR., R. E.; BANKS, P. A.; RADCLIFFE, D. E. Alachlor and metribuzin movement an dissipation in a soil profile as influenced by soil surface condition. Weed Sci., v. 38 , n. 2 , p. $589-597,1990$

LAMOREAUX, R. J.; JAIN, R.; HESS, F. D. Efficacy of dimethenamid, metolachlor and encapsulated alachlor in soil covered with crop residue. Bringhton Crop Protec. Conf. Weeds, v. 3, p. 1015-1020, 1993.

MARTINS, D. et al. Emergência em campo de dicotiledôneas infestantes em solo coberto com palha de cana-de-açúcar. Planta Daninha, v. 17, n. 1, p. 151-161, 1999.

NEGRISOLI, E. et al. Controle de plantas daninhas pelo amicarbazone aplicado na presença de palha de cana-deaçúcar. Planta Daninha, v. 25, n. 3, p. 603-611, 2007 a.
NEGRISOLI, E. et al. Associação do herbicida tebuthiuron com a cobertura de palha no controle de plantas daninhas no sistema de cana-crua. Planta Daninha, v. 25, n. 3 , p. $621-628,2007 \mathrm{~b}$.

NEGRISOLI, E. et al. Eficácia do herbicida oxyfluorfen com a cobertura de palha no controle de plantas daninhas. Planta Daninha, v. 27, n. 1, p. 197-203, 2009.

NEGRISOLI, E. Depossition and leaching of tebuthiuron on sugar cane straw applied with and without alkkyl poyglycoside adjuvnt. Journal of Environmental Science An Health. Part B, Pesticides Food Contaminants, and Agricultural Wastes, v.B40, n.1, p. $207-214,2005$

RODRIGUES, B. N.; ALMEIDA, F. S. Guia de herbicidas. 5.ed. Londrina: Grarfmake, 2005. 591 p.

SOCIEDADE BRASILEIRA DA CIÊNCIA DAS PLANTAS DANINHAS - SBCPD. Procedimentos para instalação, avaliação e análise de experimentos com herbicidas. Londrina: 1995. 42 p.

SORENSON, B. A.; SHEA, P. J.; ROETH, F. W. Effects of tillage, application time and rate on metribuzin dissipation. Weed Res., v. 31, n. 6, p. 333-345, 1991.

VELINI, E. D; NEGRISOLI, E. Controle de plantas daninhas em cana crua. In: CONGRESSO BRASILEIRO DA CIÊNCIA DAS PLANTAS DANINHAS, 22., 2000, Foz de Iguaçu. Palestras... Foz de Iguaçu: Sociedade Brasileira da Ciência das Plantas Daninhas, 2000. p. $148-164$ 